\title{
Laser assisted flow deposition: a new method to grow $\mathrm{ZnO}$
}

\author{
J. Rodrigues, M. R. N. Soares, A. J. S. Fernandes, T. Monteiro and F. M. Costa \\ Department of Physics, Laboratory I3N, University of Aveiro, Campus Universitário de Santiago, \\ 3810-193 Aveiro, Portugal
}

Zinc oxide $(\mathrm{ZnO})$ has been one of the most studied materials in the last decades. Either as bulk material, epilayers or nanostructures, this direct wide band gap semiconductor is known to possess great potential for fundamental science and modern technology applications.

In the present work, nano and micro crystals of $\mathrm{ZnO}$ were grown by the laser assisted flow deposition method at atmospheric pressure. In this new process, laser radiation impinges on the top of an extruded rod precursor, producing $\mathrm{ZnO}$ crystals by a vapour/solid (VS) mechanism. This method has proved to be very efficient, allowing high yield $\mathrm{ZnO}$ deposits at high growth rates applicable to largescale substrates [1]. ZnO structures were grown with and without the presence of silver under a power laser of $35 \mathrm{~W}$ during 3 minutes.

$\mathrm{ZnO}$ microcrystals start developing at the top of the precursor rod with a needle-like morphology, Figure 1. In this region, crystallites grow in a branching configuration from a central trunk. A secondary nucleation is observed from the termination of these needles as silver amount increases on the precursor rod. The re-nucleation and sequential growth of crystals lead to a complex threedimensional $\mathrm{ZnO}$ hierarchical architecture. The silver droplets are responsible for the re-nucleation process since they act as a catalyst for the $\mathrm{ZnO}$ growth [2].

At the silicon substrate placed $6 \mathrm{~mm}$ away from the top of precursor rod, several $\mathrm{ZnO}$ morphologies are observed: platelets resulting from bidimensional growth after needle coalescence; needles irradiating from the platelets and tetrapods, Figure 2. Among all structures tetrapods are the most frequent.

$\mathrm{X}$-ray diffraction spectra of the undoped sample and a representative one from $1 \% \mathrm{Ag}$ doped sample clearly evidence the signature of $\mathrm{ZnO}$ crystals together with metallic silver. The high crystalline quality of the $\mathrm{ZnO}$ is inferred from the narrow FWHM of the peaks corresponding to the $\mathrm{ZnO}$ wurtzite. The high crystalline quality has been corroborated by photoluminescence spectroscopy in a previous work [1].

\section{References}

1. Rodrigues, J. et al., Thin Solid Films, doi:10.1016/j.tsf.2011.10.208, 2011

2. Zhang Z. et al., J. Phys. Chem. C: 111: 17500-17505, 2007 

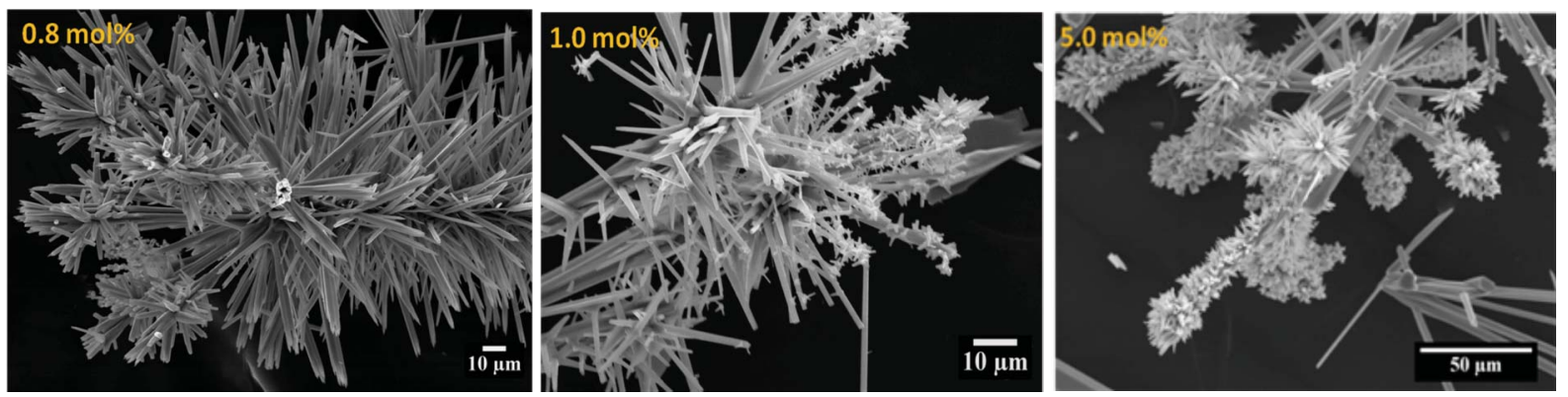

Figure 1. Scanning micrographs of Ag-doped $\mathrm{ZnO}$ structures grown on the top of the precursor rod.
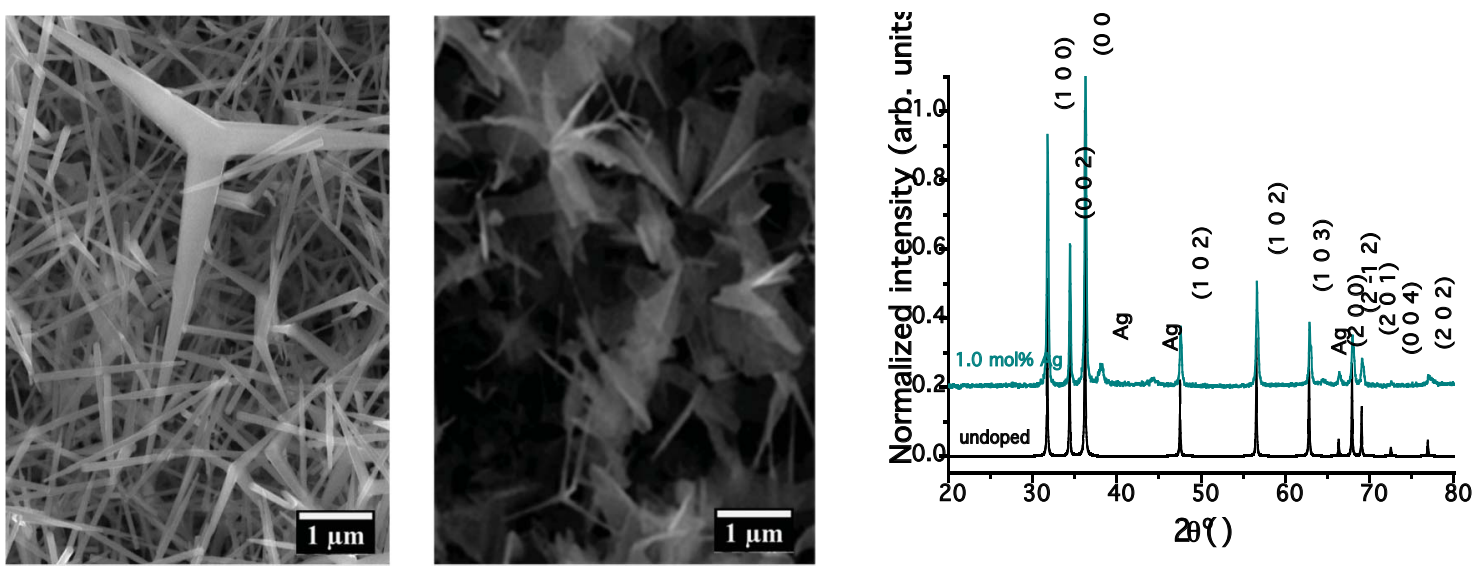

Figure 2. Scanning micrographs of Ag-doped $\mathrm{ZnO}$ structures grown on the silicon substrate surface and X-ray diffraction spectra of the undoped and 1\%Ag doped $\mathrm{ZnO}$ structures. 